TRANSACTIONS OF THE

AMERICAN MATHEMATICAL SOCIETY

Volume 361, Number 10, October 2009, Pages 5539-5554

S 0002-9947(09)04730-8

Article electronically published on May 15, 2009

\title{
A COMPARISON PRINCIPLE FOR THE COMPLEX MONGE-AMPÈRE OPERATOR IN CEGRELL'S CLASSES AND APPLICATIONS
}

\author{
NGUYEN VAN KHUE AND PHAM HOANG HIEP
}

\begin{abstract}
In this article we will first prove a result about the convergence in capacity. Next we will obtain a general decomposition theorem for complex Monge-Ampère measures which will be used to prove a comparison principle for the complex Monge-Ampère operator.
\end{abstract}

\section{INTRODUCTION}

Let $\Omega$ be a bounded hyperconvex domain in $\mathbf{C}^{n}$. Denote by $\operatorname{PSH}(\Omega)$ the set of plurisubharmonic (psh) functions on $\Omega$ and by $\mathrm{PSH}^{-}(\Omega)$ the subclass of negative functions. In [4], [5] the authors established and used the comparison principle to study the Dirichlet problem of the complex Monge-Ampère operator in PSH $\cap$ $L_{\text {loc }}^{\infty}(\Omega)$. Recently, Cegrell introduced a general class $\mathcal{E}$ of psh functions on which the complex Monge-Ampère operator $\left(d d^{c} .\right)^{n}$ is well defined. He obtained many important results of pluripotential theory in the class $\mathcal{E}$ (see [7]-9]).

The main results of our paper are Theorem 4.1 and some Xing-type comparision principles. Theorem 4.1 generalizes Lemma 5.4 in [7], Lemma 7.2 in [1] and Lemma 3.4 in [9]. For definitions of Cegrell's classes, see Section 2. After giving some preliminaries, we start in Proposition 3.1 with a comparison principle, which is analogous to a comparison principle due to Xing (Lemma 1 in [19]). It should be observed that our proof is quite different from Xing's proof, and the inequality we obtain is slightly stronger than Xing's inequality, even in the case of bounded psh functions. Using Proposition 3.1, we give in Theorem 3.5 a sufficient condition for convergence in $C_{n}$-capacity of a sequence of psh functions in the class $\mathcal{F}$. This result should be compared to Theorem 3 of [19] in which bounded psh functions were studied. Applying Theorem 3.5 we give generalizations of recent results in 11] and [12] about convergences of multipole Green functions and a criterion for pluripolarity, respectively. Section 4 focuses on Theorem 4.1 and Theorem 4.9. By applying Theorem 4.1 we give some results on Cegrell's classes. We prove in Proposition 4.4 a local estimate for the Monge-Ampère measures in terms of the Bedford-Taylor relative capacity. As an application, we give in Theorem 4.5 a decomposition result for Monge-Ampère measures, which is similar in spirit to Theorem 6.3 in [7. From Proposition 3.1 and Theorem 4.1 we obtain easily a Xing-type comparison principle for functions in classes $\mathcal{F}$ and $\mathcal{E}$.

Received by the editors December 29, 2006 and, in revised form, January 3, 2008.

2000 Mathematics Subject Classification. Primary 32W20; Secondary 32 U15.

Key words and phrases. Complex Monge-Ampère operator, plurisubharmonic function.

(C)2009 American Mathematical Society

Reverts to public domain 28 years from publication 5539 


\section{Preliminaries}

First we recall some elements of pluripotential theory that will be used throughout the paper. See also [5, 7], [8], [19, [20].

2.1. We will always denote by $\Omega$ a bounded hyperconvex domain in $\mathbf{C}^{n}$ unless otherwise stated. The $C_{n}$-capacity in the sense of Bedford and Taylor on $\Omega$ is the set function given by

$$
C_{n}(E)=C_{n}(E, \Omega)=\sup \left\{\int_{E}\left(d d^{c} u\right)^{n}: u \in \operatorname{PSH}(\Omega),-1 \leq u \leq 0\right\}
$$

for every Borel set $E$ in $\Omega$. It is proved in $[5]$ that

$$
C_{n}(E)=\int_{E}\left(d d^{c} h_{E, \Omega}^{*}\right)^{n},
$$

where $h_{E, \Omega}^{*}$ is the upper regularization of the relative extremal function $h_{E, \Omega}$ for $E$ (relative to $\Omega$ ) i.e.,

$$
h_{E, \Omega}(z)=\sup \left\{u(z): u \in \operatorname{PSH}^{-}(\Omega), u \leq-1 \text { on } E\right\} .
$$

The following concepts are taken from [19] and [20].

$*$ A sequence of functions $u_{j}$ on $\Omega$ is said to converge to a function $u$ in $C_{n}$-capacity on a set $E \subset \Omega$ if for every $\delta>0$ we have $C_{n}\left(\left\{z \in E:\left|u_{j}(z)-u(z)\right|>\delta\right\}\right) \rightarrow 0$ as $j \rightarrow \infty$.

*A family of positive measures $\left\{\mu_{\alpha}\right\}$ on $\Omega$ is said to be uniformly absolutely continuous with respect to $C_{n}$-capacity in a set $E \subset \Omega$ if for every $\epsilon>0$ there exists $\delta>0$ such that for each Borel subset $F \subset E$ with $C_{n}(\mathrm{~F})<\delta$ the inequality $\mu_{\alpha}(F)$ $<\epsilon$ holds for all $\alpha$. We write $\mu_{\alpha} \ll C_{n}$ in $E$ uniformly for $\alpha$.

2.2. The following classes of psh functions were introduced by Cegrell in [7] and [8]:

$$
\begin{aligned}
\mathcal{E}_{0} & =\mathcal{E}_{0}(\Omega)=\left\{\varphi \in \operatorname{PSH}^{-}(\Omega) \cap L^{\infty}(\Omega): \lim _{z \rightarrow \partial \Omega} \varphi(z)=0, \int_{\Omega}\left(d d^{c} \varphi\right)^{n}<+\infty\right\}, \\
\mathcal{F} & =\mathcal{F}(\Omega)=\left\{\varphi \in \operatorname{PSH}^{-}(\Omega): \exists \mathcal{E}_{0}(\Omega) \ni \varphi_{j} \searrow \varphi, \sup _{j \geq 1} \int_{\Omega}\left(d d^{c} \varphi_{j}\right)^{n}<+\infty\right\}, \\
\mathcal{E} & =\mathcal{E}(\Omega)=\left\{\varphi \in \operatorname{PSH}^{-}(\Omega): \exists \varphi_{K} \in \mathcal{F}(\Omega) \text { such that } \varphi_{K}=\varphi \text { on } K, \forall K \Subset \Omega\right\}, \\
\mathcal{E}^{a} & =\mathcal{E}^{a}(\Omega)=\left\{u \in \mathcal{E}(\Omega):\left(d d^{c} u\right)^{n}(E)=0 \forall E \text { is pluripolar in } \Omega\right\} .
\end{aligned}
$$

2.3. Let $A=\left\{\left(w_{j}, \nu_{j}\right)\right\}_{j=1, \ldots, p}$ be a finite subset of $\Omega \times \mathbf{R}^{+}$. According to Lelong (see [18]), the pluricomplex Green function with poles in $A$ is defined by

$$
g(A)(z)=\sup \left\{u(z): u \in \mathcal{L}_{A}\right\}
$$

where

$$
\mathcal{L}_{A}=\left\{u \in \mathrm{PSH}^{-}(\Omega): u(z)-\nu_{j} \log \left|z-w_{j}\right| \leq O(1) \text { as } z \rightarrow w_{j}, j=1, \ldots, p\right\} .
$$

Set

$$
\nu(A)=\sum_{j=1}^{p} \nu_{j}^{n}, \hat{A}=\left\{w_{j}\right\}_{j=1, \ldots, p} .
$$


2.4. We write $\underset{z \rightarrow \partial \Omega}{\lim }[u(z)-v(z)] \geq a$ if for every $\epsilon>0$ there exists a compact set $K$ in $\Omega$ such that

$$
u(z)-v(z) \geq a-\epsilon \text { for } z \in(\Omega \backslash K) \cap\{u>-\infty\}
$$

and

$$
v(z)=-\infty \text { for } z \in(\Omega \backslash K) \cap\{u=-\infty\} .
$$

2.5. Xing's comparison principle (see Lemma 1 in [19]). Let $\Omega$ be a bounded open subset in $\mathbf{C}^{n}$ and $u, v \in \mathrm{PSH} \cap L^{\infty}(\Omega)$ satisfy $\underset{z \rightarrow \partial \Omega}{\lim _{\rightarrow}}[u(z)-v(z)] \geq 0$. Then for any constant $r \geq 1$ and all $w_{j} \in \operatorname{PSH}(\Omega)$ with $0 \leq w_{j} \leq 1, j=1,2, \ldots, n$ we have

$$
\frac{1}{(n !)^{2}} \int_{\{u<v\}}(v-u)^{n} d d^{c} w_{1} \wedge \ldots \wedge d d^{c} w_{n}+\int_{\{u<v\}}\left(r-w_{1}\right)\left(d d^{c} v\right)^{n} \leq \int_{\{u<v\}}\left(r-w_{1}\right)\left(d d^{c} u\right)^{n} .
$$

\section{SOME CONVERGENCE THEOREMS}

In order to study the convergence of a sequence of psh functions in $C_{n}$-capacity, we start with the following.

Proposition 3.1. a) Let $u, v \in \mathcal{F}$ be such that $u \leq v$ on $\Omega$. Then for $1 \leq k \leq n$,

$$
\begin{aligned}
& \frac{1}{k !} \int_{\Omega}(v-u)^{k} d d^{c} w_{1} \wedge \ldots \wedge d d^{c} w_{n}+\int_{\Omega}\left(r-w_{1}\right)\left(d d^{c} v\right)^{k} \wedge d d^{c} w_{k+1} \wedge \ldots \wedge d d^{c} w_{n} \\
& \quad \leq \int_{\Omega}\left(r-w_{1}\right)\left(d d^{c} u\right)^{k} \wedge d d^{c} w_{k+1} \wedge \ldots \wedge d d^{c} w_{n}
\end{aligned}
$$

for all $w_{j} \in P S H(\Omega), 0 \leq w_{j} \leq 1, j=1, \ldots, k, w_{k+1}, \ldots, w_{n} \in \mathcal{F}$ and all $r \geq 1$.

b) Let $u, v \in \mathcal{E}$ be such that $u \leq v$ on $\Omega$ and $u=v$ on $\Omega \backslash K$ for some $K \Subset \Omega$. Then for $1 \leq k \leq n$,

$$
\begin{aligned}
& \frac{1}{k !} \int_{\Omega}(v-u)^{k} d d^{c} w_{1} \wedge \ldots \wedge d d^{c} w_{n}+\int_{\Omega}\left(r-w_{1}\right)\left(d d^{c} v\right)^{k} \wedge d d^{c} w_{k+1} \wedge \ldots \wedge d d^{c} w_{n} \\
& \quad \leq \int_{\Omega}\left(r-w_{1}\right)\left(d d^{c} u\right)^{k} \wedge d d^{c} w_{k+1} \wedge \ldots \wedge d d^{c} w_{n},
\end{aligned}
$$

for all $w_{j} \in P S H(\Omega), 0 \leq w_{j} \leq 1, j=1, \ldots, k, w_{k+1}, \ldots, w_{n} \in \mathcal{E}$ and all $r \geq 1$.

We proceed through some lemmas.

Lemma 3.2. Let $u, v \in P S H \cap L^{\infty}(\Omega)$ be such that $u \leq v$ on $\Omega$ and $\lim _{z \rightarrow \partial \Omega}[u(z)-v(z)]=0$. Then

$$
\int_{\Omega}(v-u)^{k} d d^{c} w \wedge T \leq k \int_{\Omega}(1-w)(v-u)^{k-1} d d^{c} u \wedge T,
$$

for all $w \in P S H(\Omega), 0 \leq w \leq 1$ and all positive closed currents $T$. 
Proof. First, assume $u, v \in \operatorname{PSH} \cap L^{\infty}(\Omega), u \leq v$ on $\Omega$ and $u=v$ on $\Omega \backslash K, K \Subset \Omega$. Then, using the Stokes formula we obtain

$$
\begin{aligned}
\int_{\Omega}(v-u)^{k} d d^{c} w \wedge T= & \int_{\Omega}(v-u)^{k} d d^{c}(w-1) \wedge T \\
= & \int_{\Omega}(w-1) d d^{c}(v-u)^{k} \wedge T \\
= & -k(k-1) \int_{\Omega}(1-w) d(v-u) \wedge d^{c}(v-u) \wedge T \\
& +k \int_{\Omega}(1-w)(v-u)^{k-1} d d^{c}(u-v) \wedge T \\
\leq & k \int_{\Omega}(1-w)(v-u)^{k-1} d d^{c}(u-v) \wedge T \\
\leq & k \int_{\Omega}(1-w)(v-u)^{k-1} d d^{c} u \wedge T .
\end{aligned}
$$

In the general case, for each $\epsilon>0$ we set $v_{\epsilon}=\max (u, v-\epsilon)$. Then $v_{\epsilon} \nearrow v$ on $\Omega$, $v_{\epsilon} \geq u$ on $\Omega$ and $v_{\epsilon}=u$ on $\Omega \backslash K$ for some $K \Subset \Omega$. Hence

$$
\int_{\Omega}\left(v_{\epsilon}-u\right)^{k} d d^{c} w \wedge T \leq k \int_{\Omega}(1-w)\left(v_{\epsilon}-u\right)^{k-1} d d^{c} u \wedge T .
$$

Since $0 \leq v_{\epsilon}-u \nearrow v-u$ as $\epsilon \searrow 0$, letting $\epsilon \searrow 0$ we get

$$
\int_{\Omega}(v-u)^{k} d d^{c} w \wedge T \leq k \int_{\Omega}(1-w)(v-u)^{k-1} d d^{c} u \wedge T .
$$

Lemma 3.3. Let $u, v \in P S H \cap L^{\infty}(\Omega)$ be such that $u \leq v$ on $\Omega$ and $\lim _{z \rightarrow \partial \Omega}[u(z)-v(z)]=0$. Then for $1 \leq k \leq n$,

$$
\begin{aligned}
& \frac{1}{k !} \int_{\Omega}(v-u)^{k} d d^{c} w_{1} \wedge \ldots \wedge d d^{c} w_{n}+\int_{\Omega}\left(r-w_{1}\right)\left(d d^{c} v\right)^{k} \wedge d d^{c} w_{k+1} \wedge \ldots \wedge d d^{c} w_{n} \\
& \quad \leq \int_{\Omega}\left(r-w_{1}\right)\left(d d^{c} u\right)^{k} \wedge d d^{c} w_{k+1} \wedge \ldots \wedge d d^{c} w_{n}
\end{aligned}
$$

for all $w_{1}, \ldots, w_{k} \in P S H(\Omega), 0 \leq w_{j} \leq 1 \forall j=1, \ldots, k, w_{k+1}, \ldots, w_{n} \in \mathcal{E}$ and all $r \geq 1$.

Proof. To simplify the notation we set

$$
T=d d^{c} w_{k+1} \wedge \ldots \wedge d d^{c} w_{n}
$$


First, assume that $u, v \in \operatorname{PSH} \cap L^{\infty}(\Omega), u \leq v$ on $\Omega$, and $u=v$ on $\Omega \backslash K, K \Subset \Omega$. Using Lemma 3.2 we get

$$
\begin{aligned}
& \int_{\Omega}(v-u)^{k} d d^{c} w_{1} \wedge \ldots \wedge d d^{c} w_{n} \leq k \int_{\Omega}(v-u)^{k-1} d d^{c} w_{1} \wedge \ldots \wedge d d^{c} w_{k-1} \wedge d d^{c} u \wedge T \\
& \quad \leq k ! \int_{\Omega}(v-u) d d^{c} w_{1} \wedge\left(d d^{c} u\right)^{k-1} \wedge T \\
& \quad \leq k ! \int_{\Omega}(v-u) d d^{c} w_{1} \wedge\left[\sum_{i=0}^{k-1}\left(d d^{c} u\right)^{i} \wedge\left(d d^{c} v\right)^{k-i-1}\right] \wedge T \\
& =k ! \int_{\Omega}\left(w_{1}-r\right) d d^{c}(v-u) \wedge\left[\sum_{i=0}^{k-1}\left(d d^{c} u\right)^{i} \wedge\left(d d^{c} v\right)^{k-i-1}\right] \wedge T \\
& =k ! \int_{\Omega}\left(r-w_{1}\right) d d^{c}(u-v) \wedge\left[\sum_{i=0}^{k-1}\left(d d^{c} u\right)^{i} \wedge\left(d d^{c} v\right)^{k-i-1}\right] \wedge T \\
& =k ! \int_{\Omega}\left(r-w_{1}\right)\left[\left(d d^{c} u\right)^{k}-\left(d d^{c} v\right)^{k}\right] \wedge T .
\end{aligned}
$$

In the general case, for each $\epsilon>0$ we put $v_{\epsilon}=\max (u, v-\epsilon)$. Then $v_{\epsilon} \nearrow v$ on $\Omega$, $v_{\epsilon} \geq u$ on $\Omega$ and $v_{\epsilon}=u$ on $\Omega \backslash K$ for some $K \Subset \Omega$. Hence

$$
\begin{aligned}
& \frac{1}{k !} \int_{\Omega}\left(v_{\epsilon}-u\right)^{k} d d^{c} w_{1} \wedge \ldots \wedge d d^{c} w_{n}+\int_{\Omega}\left(r-w_{1}\right)\left(d d^{c} v_{\epsilon}\right)^{k} \wedge T \\
& \quad \leq \int_{\Omega}\left(r-w_{1}\right)\left(d d^{c} u\right)^{k} \wedge T .
\end{aligned}
$$

Observe that $0 \leq v_{\epsilon}-u \nearrow v-u$ and $\left(d d^{c} v_{\epsilon}\right)^{k} \wedge T \rightarrow\left(d d^{c} v\right)^{k} \wedge T$ weakly as $\epsilon \searrow 0$, $r-w_{1}$ is lower semicontinuous, and by letting $\epsilon \searrow 0$ we have

$$
\begin{aligned}
& \frac{1}{k !} \int_{\Omega}(v-u)^{k} d d^{c} w_{1} \wedge \ldots \wedge d d^{c} w_{n}+\int_{\Omega}\left(r-w_{1}\right)\left(d d^{c} v\right)^{k} \wedge T \\
& \quad \leq \int_{\Omega}\left(r-w_{1}\right)\left(d d^{c} u\right)^{k} \wedge T .
\end{aligned}
$$

The proof is finished.

Proof of Proposition 3.1. a) Let $\mathcal{E}_{0} \ni u_{j} \searrow u$ and $\mathcal{E}_{0} \ni v_{j} \searrow v$ as in the definition of $\mathcal{F}$. Replacing $v_{j}$ by $\max \left(u_{j}, v_{j}\right)$ we may assume that $u_{j} \leq v_{j}$ for $j \geq 1$. By Lemma 3.3 we have

$$
\begin{aligned}
& \frac{1}{k !} \int_{\Omega}\left(v_{j}-u_{t}\right)^{k} d d^{c} w_{1} \wedge \ldots \wedge d d^{c} w_{n}+\int_{\Omega}\left(r-w_{1}\right)\left(d d^{c} v_{j}\right)^{k} \wedge d d^{c} w_{k+1} \wedge \ldots \wedge d d^{c} w_{n} \\
& \quad \leq \int_{\Omega}\left(r-w_{1}\right)\left(d d^{c} u_{t}\right)^{k} \wedge d d^{c} w_{k+1} \wedge \ldots \wedge d d^{c} w_{n}
\end{aligned}
$$


for $t \geq j \geq 1$. By Proposition 5.1 in $[\underline{8}$, letting $t \rightarrow \infty$ in the above inequality, we have

$$
\begin{aligned}
& \frac{1}{k !} \int_{\Omega}\left(v_{j}-u\right)^{k} d d^{c} w_{1} \wedge \ldots \wedge d d^{c} w_{n}+\int_{\Omega}\left(r-w_{1}\right)\left(d d^{c} v_{j}\right)^{k} \wedge d d^{c} w_{k+1} \wedge \ldots \wedge d d^{c} w_{n} \\
& \quad \leq \int_{\Omega}\left(r-w_{1}\right)\left(d d^{c} u\right)^{k} \wedge d d^{c} w_{k+1} \wedge \ldots \wedge d d^{c} w_{n}
\end{aligned}
$$

for $j \geq 1$. Next letting $j \rightarrow \infty$ again by Proposition 5.1 in [8] we get the desired conclusion.

b) Let $G, W$ be open sets such that $K \Subset G \Subset W \Subset \Omega$. According to the remark after Definition 4.6 in [8] we can choose a function $\tilde{v} \in \mathcal{F}$ such that $\tilde{v} \geq v$ and $\tilde{v}=v$ on $W$. Set

$$
\tilde{u}= \begin{cases}u & \text { on } G \\ \tilde{v} & \text { on } \Omega \backslash G .\end{cases}
$$

Since $u=v=\tilde{v}$ on $W \backslash K$ we have $\tilde{u} \in \operatorname{PSH}^{-}(\Omega)$. It is easy to see that $\tilde{u} \in \mathcal{F}$, $\tilde{u} \leq \tilde{v}$ and $\tilde{u}=u$ on $W$. By a) we have

$$
\begin{aligned}
& \frac{1}{k !} \int_{\Omega}(\tilde{v}-\tilde{u})^{k} d d^{c} w_{1} \wedge \ldots \wedge d d^{c} w_{n}+\int_{\Omega}\left(r-w_{1}\right)\left(d d^{c} \tilde{v}\right)^{k} \wedge d d^{c} w_{k+1} \wedge \ldots \wedge d d^{c} w_{n} \\
& \quad \leq \int_{\Omega}\left(r-w_{1}\right)\left(d d^{c} \tilde{u}\right)^{k} \wedge d d^{c} w_{k+1} \wedge \ldots \wedge d d^{c} w_{n}
\end{aligned}
$$

Since $\tilde{u}=\tilde{v}$ on $\Omega \backslash G$ we have

$$
\begin{aligned}
& \frac{1}{k !} \int_{W}(\tilde{v}-\tilde{u})^{k} d d^{c} w_{1} \wedge \ldots \wedge d d^{c} w_{n}+\int_{W}\left(r-w_{1}\right)\left(d d^{c} \tilde{v}\right)^{k} \wedge d d^{c} w_{k+1} \wedge \ldots \wedge d d^{c} w_{n} \\
& \quad \leq \int_{W}\left(r-w_{1}\right)\left(d d^{c} \tilde{u}\right)^{k} \wedge d d^{c} w_{k+1} \wedge \ldots \wedge d d^{c} w_{n} .
\end{aligned}
$$

Since $\tilde{u}=u, \tilde{v}=v$ on $W$ and $u=v$ on $\Omega \backslash K$ we obtain

$$
\begin{aligned}
& \frac{1}{k !} \int_{\Omega}(v-u)^{k} d d^{c} w_{1} \wedge \ldots \wedge d d^{c} w_{n}+\int_{\Omega}\left(r-w_{1}\right)\left(d d^{c} v\right)^{k} \wedge d d^{c} w_{k+1} \wedge \ldots \wedge d d^{c} w_{n} \\
& \quad \leq \int_{\Omega}\left(r-w_{1}\right)\left(d d^{c} u\right)^{k} \wedge d d^{c} w_{k+1} \wedge \ldots \wedge d d^{c} w_{n} .
\end{aligned}
$$

Proposition 3.4. Let $u, v \in \mathcal{F}$ be such that $u \leq v$ on $\Omega$. Then

$$
\frac{1}{n !} \int_{\Omega}(v-u)^{n} d d^{c} w_{1} \wedge \ldots \wedge d d^{c} w_{n} \leq \int_{\Omega}\left(-w_{1}\right)\left[\left(d d^{c} u\right)^{n}-\left(d d^{c} v\right)^{n}\right]
$$

for all $w_{j} \in P S H(\Omega),-1 \leq w_{j} \leq 0, j=1, \ldots, n$.

Proof. The proposition follows from Proposition 3.1 with $k=n, r=1$ and with $w_{j}$ replaced by $w_{j}+1$. 
Theorem 3.5. Let $u, u_{j} \in \mathcal{F}$ be such that $u_{j} \leq u$ for $j \geq 1$. Assume that

$$
\sup _{j \geq 1} \int_{\Omega}\left(d d^{c} u_{j}\right)^{n}<+\infty
$$

and $\left\|\left(d d^{c} u_{j}\right)^{n}-\left(d d^{c} u\right)^{n}\right\|_{E} \rightarrow 0$ as $j \rightarrow \infty$ for all $E \Subset \Omega$. Then $u_{j} \rightarrow u$ in $C_{n}$-capacity on every $E \Subset \Omega$ as $j \rightarrow \infty$.

Proof. Let $\Omega^{\prime} \Subset \Omega$ and $\delta>0$. Put

$$
A_{j}=\left\{z \in \overline{\Omega^{\prime}}:\left|u_{j}-u\right| \geq \delta\right\}=\left\{z \in \overline{\Omega^{\prime}}: u-u_{j} \geq \delta\right\}
$$

We prove that $C_{n}\left(A_{j}\right) \rightarrow 0$ as $j \rightarrow \infty$. Given $\epsilon>0$, by quasicontinuity of $u$ and $u_{j}$, there is an open set $G$ in $\Omega$ such that $C_{n}(G)<\epsilon$, and $\left.u_{j}\right|_{\Omega \backslash G},\left.u\right|_{\Omega \backslash G}$ are continuous. We have

$$
A_{j}=B_{j} \cup\left\{z \in G: u-u_{j} \geq \delta\right\},
$$

where $B_{j}=\left\{z \in \overline{\Omega^{\prime}} \backslash G: u-u_{j} \geq \delta\right\}$ are compact sets in $\Omega$ and

$$
\varlimsup_{j \rightarrow \infty} C_{n}\left(A_{j}\right) \leq \varlimsup_{j \rightarrow \infty} C_{n}\left(B_{j}\right)+\epsilon .
$$

We claim that $\lim _{j \rightarrow \infty} C_{n}\left(B_{j}\right)=0$. By Proposition 3.4 we have

$$
\begin{aligned}
C_{n}\left(B_{j}\right) & =\int_{B_{j}}\left(d d^{c} h_{B_{j}}^{*}\right)^{n} \\
& \leq \frac{1}{\delta^{n}} \int_{B_{j}}\left(u-u_{j}\right)^{n}\left(d d^{c} h_{B_{j}}^{*}\right)^{n} \\
& \leq \frac{n !}{\delta^{n}} \int_{\Omega}\left(-h_{B_{j}}^{*}\right)\left[\left(d d^{c} u_{j}\right)^{n}-\left(d d^{c} u\right)^{n}\right] \\
& \leq \frac{n !}{\delta^{n}}\left\{\left\|\left(d d^{c} u_{j}\right)^{n}-\left(d d^{c} u\right)^{n}\right\|_{K}+\int_{\Omega \backslash K}\left(-h_{\Omega^{\prime}}\right)\left[\left(d d^{c} u_{j}\right)^{n}+\left(d d^{c} u\right)^{n}\right]\right\} \\
& \leq \frac{n !}{\delta^{n}}\left\{\left\|\left(d d^{c} u_{j}\right)^{n}-\left(d d^{c} u\right)^{n}\right\|_{K}+\sup _{\Omega \backslash K}\left|h_{\Omega^{\prime}}\right|\left[\sup _{j \geq 1} \int_{\Omega}\left(d d^{c} u_{j}\right)^{n}+\int_{\Omega}\left(d d^{c} u\right)^{n}\right]\right\} .
\end{aligned}
$$

As $\lim _{z \rightarrow \partial \Omega} h_{\Omega^{\prime}}(z)=0$ there exists $K \Subset \Omega$ such that

$$
\frac{n !}{\delta^{n}} \sup _{\Omega \backslash K}\left|h_{\Omega^{\prime}}\right|\left[\sup _{j \geq 1} \int_{\Omega}\left(d d^{c} u_{j}\right)^{n}+\int_{\Omega}\left(d d^{c} u\right)^{n}\right]<\epsilon .
$$

By the hypothesis,

$$
\frac{n !}{\delta^{n}}\left\|\left(d d^{c} u_{j}\right)^{n}-\left(d d^{c} u\right)^{n}\right\|_{K}<\epsilon \text { for } j>j_{0} .
$$

Thus

$$
C_{n}\left(B_{j}\right)<2 \epsilon \text { for } j>j_{0} .
$$

This proves the claim and hence the theorem. 
As an application of Theorem 3.5 we have the following.

Proposition 3.6. Let $g\left(A_{j}\right)$ be multipolar Green functions on $\Omega$ such that

$$
\hat{A}_{j}=\left\{w_{1}^{j}, \ldots, w_{p_{j}}^{j}\right\} \rightarrow \partial \Omega \text { and } \sup _{j \geq 1} \nu\left(A_{j}\right)=\sup _{j \geq 1} \sum_{k=1}^{p_{j}}\left(\nu_{k}^{j}\right)^{n}<+\infty .
$$

Then $g\left(A_{j}\right) \rightarrow 0$ as $j \rightarrow \infty$ in $C_{n}$-capacity.

Proof. By the hypothesis we have

$$
\sup _{j \geq 1}\left(d d^{c} g\left(A_{j}\right)\right)^{n}(\Omega)=\sup _{j \geq 1} \nu\left(A_{j}\right)<+\infty
$$

and

$$
\left\|\left(d d^{c} g\left(A_{j}\right)\right)^{n}\right\|_{K} \rightarrow 0 \text { as } j \rightarrow \infty \text { for all } K \Subset \Omega .
$$

Theorem 3.5 implies that $g\left(A_{j}\right) \rightarrow 0$ as $j \rightarrow \infty$ in $C_{n}$-capacity.

This section ends with a criterion for pluripolarity.

Theorem 3.7. Let $u_{j} \in \mathcal{F}$ be such that $\sup _{j \geq 1} \int_{\Omega}\left(d d^{c} u_{j}\right)^{n}<+\infty$.

Then there is a constant $A>0$ such that

i) $\left(\varlimsup_{j \rightarrow \infty} u_{j}\right)^{*} \in \mathcal{F}$,

ii) $C_{n}\left(\left\{z \in \Omega:\left(\varlimsup_{j \rightarrow \infty} u_{j}\right)^{*}(z)<-t\right\}\right) \leq \frac{A}{t^{n}}$,

iii) $\left\{z \in \Omega: \lim _{j \rightarrow \infty} u_{j}(z)=-\infty\right\}$ is pluripolar.

Proof. i) For each $j \geq 1$ put $v_{j}=\sup \left\{u_{j}, u_{j+1}, \ldots\right\}$. By [8], $v_{j}^{*} \in \mathcal{F}$ and

$$
\sup _{j \geq 1} \int_{\Omega}\left(d d^{c} v_{j}^{*}\right)^{n} \leq \sup _{j \geq 1} \int_{\Omega}\left(d d^{c} u_{j}\right)^{n}<+\infty .
$$

By [8] we have $v_{j}^{*} \searrow v \in \mathcal{F}$.

ii) By Proposition 3.1 in [10] we have

$$
C_{n}\left\{z \in \Omega:\left(\varlimsup_{j \rightarrow \infty} u_{j}\right)^{*}(z)<-t\right\}=C_{n}\{z \in \Omega: v(z)<-t\} \leq \frac{2^{n} \int_{\Omega}\left(d d^{c} v\right)^{n}}{t^{n}}=\frac{A}{t^{n}},
$$

where $A=2^{n} \int_{\Omega}\left(d d^{c} v\right)^{n}$.

iii) According to [5] we have

$$
C_{n}\left\{z \in \Omega: \lim _{j \rightarrow \infty} u_{j}(z)=-\infty\right\}=C_{n}\{z \in \Omega: v(z)=-\infty\}=0 .
$$

Remark. Theorem 3.7 in the case where the $u_{j}$ are multipole Green functions was proved by D. Coman, N. Levenberg and A. Poletsky in Theorem 4.1 of 12 .

\section{Some properties of Cegrell's classes and applications}

In this section, first we prove the following.

Theorem 4.1. Let $u, u_{1}, \ldots, u_{n-1} \in \mathcal{E}, v \in P S H^{-}(\Omega)$ and $T=d d^{c} u_{1} \wedge \ldots \wedge d d^{c} u_{n-1}$. Then

$$
\left.d d^{c} \max (u, v) \wedge T\right|_{\{u>v\}}=\left.d d^{c} u \wedge T\right|_{\{u>v\}} .
$$

We need the following well-known fact. 
Lemma 4.2. Let $\mu$ be a Borel measure on $\Omega$ and $f: \Omega \rightarrow \mathbf{R}$ a measurable function on $\Omega$. The following are equivalent:

i) $\mu(E)=0$ for all Borel sets $E \subset\{f \neq 0\}$,

ii) $\int_{E} f d \mu=0$ for all Borel sets $E$ in $\Omega$.

Proof. i) $\Rightarrow$ ii) follows from:

$$
\int_{E} f d \mu=\int_{E \backslash\{f=0\}} f d \mu+\int_{E \cap\{f=0\}} f d \mu=0 .
$$

ii) $\Rightarrow$ i). It suffices to show that $\mu=0$ on every $X_{\delta}=\{f>\delta>0\}$. By the Hahn decomposition theorem, there exist measurable subsets $X_{\delta}^{+}$and $X_{\delta}^{-}$of $X_{\delta}$ such that $X_{\delta}=X_{\delta}^{+} \cup X_{\delta}^{-}, X_{\delta}^{+} \cap X_{\delta}^{-}=\emptyset$ and $\mu \geq 0$ on $X_{\delta}^{+}, \mu \leq 0$ on $X_{\delta}^{-}$. We have

$$
\left\{\begin{array}{l}
\delta \mu\left(X_{\delta}^{+}\right) \leq \int_{X_{\delta}^{+}} f d \mu=0, \\
\delta \mu\left(X_{\delta}^{-}\right) \geq \int_{X_{\delta}^{-}} f d \mu=0 .
\end{array}\right.
$$

Hence, $\mu\left(X_{\delta}^{+}\right)=\mu\left(X_{\delta}^{-}\right)=0$. Therefore, we have $\mu=0$ on $X_{\delta}$.

Proof of Theorem 4.1. a) First we prove the proposition for $v \equiv a<0$. According to the remark following Definition 4.6 in [ 8 , without loss of generality we may assume that $u, u_{1}, \ldots, u_{n-1} \in \mathcal{F}$. Using Theorem 2.1 in $[8]$ we can find

$$
\mathcal{E}_{0} \cap C(\bar{\Omega}) \ni u^{j} \searrow u, \mathcal{E}_{0} \cap C(\bar{\Omega}) \ni u_{k}^{j} \searrow u_{k}, k=1, \ldots, n-1 .
$$

Since $\left\{u^{j}>a\right\}$ is open we have

$$
\left.d d^{c} \max \left(u^{j}, a\right) \wedge T_{j}\right|_{\left\{u^{j}>a\right\}}=\left.d d^{c} u^{j} \wedge T_{j}\right|_{\left\{u^{j}>a\right\}} .
$$

Thus from the inclusion $\{u>a\} \subset\left\{u^{j}>a\right\}$ we obtain

$$
\left.d d^{c} \max \left(u^{j}, a\right) \wedge T_{j}\right|_{\{u>a\}}=\left.d d^{c} u^{j} \wedge T_{j}\right|_{\{u>a\}},
$$

where $T_{j}=d d^{c} u_{1}^{j} \wedge \ldots \wedge d d^{c} u_{n-1}^{j}$. By Corollary 5.2 in [8], it follows that

$$
\begin{aligned}
& \max (u-a, 0) d d^{c} \max \left(u^{j}, a\right) \wedge T_{j} \rightarrow \max (u-a, 0) d d^{c} \max (u, a) \wedge T, \\
& \max (u-a, 0) d d^{c} u^{j} \wedge T_{j} \rightarrow \max (u-a, 0) d d^{c} u \wedge T .
\end{aligned}
$$

Hence

$$
\max (u-a, 0)\left[d d^{c} \max (u, a) \wedge T-d d^{c} u \wedge T\right]=0 .
$$

Using Lemma 4.2 we have

$$
d d^{c} \max (u, a) \wedge T=d d^{c} u \wedge T \text { on }\{u>a\} .
$$

b) Assume that $v \in P S H^{-}(\Omega)$. Since $\{u>v\}=\bigcup_{a \in \mathbf{Q}^{-}}\{u>a>v\}$, it suffices to show that

$$
d d^{c} \max (u, v) \wedge T=d d^{c} u \wedge T \text { on }\{u>a>v\}
$$

for all $a \in \mathbf{Q}^{-}$, the set of negative rational numbers. Since $\max (u, v) \in \mathcal{E}$, by a) we have

$$
\begin{aligned}
\left.d d^{c} \max (u, v) \wedge T\right|_{\{\max (u, v)>a\}} & =\left.d d^{c} \max (\max (u, v), a) \wedge T\right|_{\{\max (u, v)>a\}} \\
& =\left.d d^{c} \max (u, v, a) \wedge T\right|_{\{\max (u, v)>a\}}, \\
\left.d d^{c} u \wedge T\right|_{\{u>a\}} & =\left.d d^{c} \max (u, a) \wedge T\right|_{\{u>a\}} .
\end{aligned}
$$


Since $\max (u, v, a)=\max (u, a)$ on the open set $\{a>v\}$, we have

$$
\left.d d^{c} \max (u, v, a) \wedge T\right|_{\{a>v\}}=\left.d d^{c} \max (u, a) \wedge T\right|_{\{a>v\}} .
$$

Since $\{u>a>v\} \subset\{u>a\},\{a>v\},\{\max (u, v)>a\}$ and (1), (2), (3) we have

$$
\left.d d^{c} \max (u, v) \wedge T\right|_{\{u>a>v\}}=\left.d d^{c} u \wedge T\right|_{\{u>a>v\}} .
$$

The next result is an analogue of an inequality due to Demailly in [14.

Proposition 4.3. a) Let $u, v \in \mathcal{E}$ be such that $\left(d d^{c} u\right)^{n}(\{u=v=-\infty\})=0$. Then

$$
\left(d d^{c} \max (u, v)\right)^{n} \geq 1_{\{u \geq v\}}\left(d d^{c} u\right)^{n}+1_{\{u<v\}}\left(d d^{c} v\right)^{n},
$$

where $1_{E}$ denotes the characteristic function of $E$.

b) Let $\mu$ be a positive measure which vanishes on all pluripolar subsets of $\Omega$. Suppose $u, v \in \mathcal{E}$ such that $\left(d d^{c} u\right)^{n} \geq \mu,\left(d d^{c} v\right)^{n} \geq \mu$. Then $\left(d d^{c} \max (u, v)\right)^{n} \geq \mu$.

Proof. a) For each $\epsilon>0$ put $A_{\epsilon}=\{u=v-\epsilon\} \backslash\{u=v=-\infty\}$. Since $A_{\epsilon} \cap A_{\delta}=\emptyset$ for $\epsilon \neq \delta$ there exists $\epsilon_{j} \searrow 0$ such that $\left(d d^{c} u\right)^{n}\left(A_{\epsilon_{j}}\right)=0$ for $j \geq 1$. On the other hand, since $\left(d d^{c} u\right)^{n}(\{u=v=-\infty\})=0$ we have $\left(d d^{c} u\right)^{n}\left(\left\{u=v-\epsilon_{j}\right\}\right)=0$ for $j \geq 1$. By Theorem 4.1 it follows that

$$
\begin{aligned}
\left(d d^{c} \max \left(u, v-\epsilon_{j}\right)\right)^{n} \geq & \left.\left(d d^{c} \max \left(u, v-\epsilon_{j}\right)\right)^{n}\right|_{\left\{u>v-\epsilon_{j}\right\}} \\
& +\left.\left(d d^{c} \max \left(u, v-\epsilon_{j}\right)\right)^{n}\right|_{\left\{u<v-\epsilon_{j}\right\}} \\
= & \left.\left(d d^{c} u\right)^{n}\right|_{\left\{u \geq v-\epsilon_{j}\right\}}+\left.\left(d d^{c} v\right)^{n}\right|_{\left\{u<v-\epsilon_{j}\right\}} \\
= & 1_{\left\{u \geq v-\epsilon_{j}\right\}}\left(d d^{c} u\right)^{n}+1_{\left\{u<v-\epsilon_{j}\right\}}\left(d d^{c} v\right)^{n} \\
\geq & 1_{\{u \geq v\}}\left(d d^{c} u\right)^{n}+1_{\left\{u<v-\epsilon_{j}\right\}}\left(d d^{c} v\right)^{n} .
\end{aligned}
$$

Letting $j \rightarrow \infty$ and by the remark following Theorem 5.15 in 8 we get

$$
\left(d d^{c} \max (u, v)\right)^{n} \geq 1_{\{u \geq v\}}\left(d d^{c} u\right)^{n}+1_{\{u<v\}}\left(d d^{c} v\right)^{n},
$$

because $\max \left(u, v-\epsilon_{j}\right) \nearrow \max (u, v)$ and $1_{\left\{u<v-\epsilon_{j}\right\}} \nearrow 1_{\{u<v\}}$ as $j \rightarrow \infty$.

b) The argument is the same as a).

Proposition 4.4. Let $u_{1}, \ldots, u_{k} \in P S H(\Omega) \cap L^{\infty}(\Omega)$ and $u_{k+1}, \ldots, u_{n} \in \mathcal{E}$. Then i) $\int_{B} d d^{c} u_{1} \wedge \ldots \wedge d d^{c} u_{n}=O\left(\left(C_{n}(B)\right)^{\frac{k}{n}}\right)$ for all Borel sets $B \subset \Omega^{\prime} \Subset \Omega$.

ii) $\int_{B(a, r)} d d^{c} u_{1} \wedge \ldots \wedge d d^{c} u_{n}=o\left(\left(C_{n}(B(a, r))\right)^{\frac{k}{n}}\right)$ as $r \rightarrow 0$ for all $a \in \Omega$, where $B(a, r)=\left\{z \in \mathbf{C}^{n}:|z-a|<r\right\}$.

Proof. We may assume that $0 \leq u_{j} \leq 1$ for $j=1, \ldots, k$. On the other hand, by the remark following Definition 4.6 in [8] we again may assume that $u_{k+1}, \ldots, u_{n} \in \mathcal{F}$. 
i) For each open set $B \Subset \Omega$, applying Proposition 3.1 and Corollary 5.6 in $[8$ we get

$$
\begin{aligned}
& \int_{B} d d^{c} u_{1} \wedge \ldots \wedge d d^{c} u_{n}=\int_{B}\left(-h_{B}^{*}\right)^{k} d d^{c} u_{1} \wedge \ldots \wedge d d^{c} u_{n} \\
& \leq \int_{\Omega}\left(-h_{B}^{*}\right)^{k} d d^{c} u_{1} \wedge \ldots \wedge d d^{c} u_{n} \\
& \leq k ! \int_{\Omega}\left(1-u_{1}\right)\left(d d^{c} h_{B}^{*}\right)^{k} \wedge d d^{c} u_{k+1} \wedge \ldots \wedge d d^{c} u_{n} \\
& \leq k ! \int_{\Omega}\left(d d^{c} h_{B}^{*}\right)^{k} \wedge d d^{c} u_{k+1} \wedge \ldots \wedge d d^{c} u_{n} \\
& \leq k !\left[\int_{\Omega}\left(d d^{c} h_{B}^{*}\right)^{n}\right]^{\frac{k}{n}}\left[\int_{\Omega}\left(d d^{c} u_{k+1}\right)^{n}\right]^{\frac{1}{n}} \ldots\left[\int_{\Omega}\left(d d^{c} u_{n}\right)^{n}\right]^{\frac{1}{n}} \\
& \leq k !\left[\int_{\Omega}\left(d d^{c} u_{k+1}\right)^{n}\right]^{\frac{1}{n}} \ldots\left[\int_{\Omega}\left(d d^{c} u_{n}\right)^{n}\right]^{\frac{1}{n}} \cdot\left[C_{n}(B)\right]^{\frac{k}{n}} \\
& \leq \text { constants. }\left[C_{n}(B)\right]^{\frac{k}{n}} \text {. }
\end{aligned}
$$

Hence

$$
\int_{B} d d^{c} u_{1} \wedge \ldots \wedge d d^{c} u_{n} \leq \text { constants. }\left[C_{n}(B)\right]^{\frac{k}{n}}
$$

for all Borel sets $B \subset \Omega$.

ii) By Proposition 3.1 we have

$$
\begin{aligned}
\int_{\Omega}(-\varphi)^{k} d d^{c} u_{1} \wedge \ldots \wedge d d^{c} u_{n} & \leq k ! \int_{\Omega}\left(1-u_{1}\right)\left(d d^{c} \varphi\right)^{k} \wedge d d^{c} u_{k+1} \wedge \ldots \wedge d d^{c} u_{n} \\
& \leq k ! \int_{\Omega}\left(d d^{c} \varphi\right)^{k} \wedge d d^{c} u_{k+1} \wedge \ldots \wedge d d^{c} u_{n}<+\infty
\end{aligned}
$$

Hence $(-\varphi)^{k} \in L_{1}\left(d d^{c} u_{1} \wedge \ldots \wedge d d^{c} u_{n}\right)$ for all $\varphi \in \mathcal{F}(\Omega)$. Given $a \in \Omega$ let $r_{0}, R_{0}$ be such that $B\left(a, r_{0}\right) \Subset \Omega \Subset B\left(a, R_{0}\right)$. Then

$$
\log \frac{|z-a|}{R_{0}} \leq g_{a}(z) \leq \log \frac{|z-a|}{r_{0}}
$$

for all $z \in \Omega$, where $g_{a}$ denotes the Green function of $\Omega$ with pole at $a$. Since $\left(-g_{a}\right)^{k} \in L_{1}\left(d d^{c} u_{1} \wedge \ldots \wedge d d^{c} u_{n}\right)$, it follows that

$$
\int_{B(a, r)}\left(-g_{a}\right)^{k} d d^{c} u_{1} \wedge \ldots \wedge d d^{c} u_{n} \rightarrow 0 \text { as } r \rightarrow 0 .
$$

Hence

$$
\left(\log r_{0}-\log r\right)^{k} \int_{B(a, r)} d d^{c} u_{1} \wedge \ldots \wedge d d^{c} u_{n} \leq \int_{B(a, r)}\left(-g_{a}\right)^{k} d d^{c} u_{1} \wedge \ldots \wedge d d^{c} u_{n} \rightarrow 0
$$


as $r \rightarrow 0$. This means that

$$
\int_{B(a, r)} d d^{c} u_{1} \wedge \ldots \wedge d d^{c} u_{n}=o\left(\left(\frac{1}{\log r_{0}-\log r}\right)^{k}\right) \text { as } r \rightarrow 0 .
$$

Combining this with the inequality

$C_{n}(B(a, r), \Omega) \geq C_{n}\left(B(a, r), B\left(a, R_{0}\right)\right)=\left(\frac{1}{\log R_{0}-\log r}\right)^{n}=O\left(\left(\frac{1}{\left.\log r_{0}-\log r\right)^{n}}\right)\right.$,

we get

$$
\int_{B(a, r)} d d^{c} u_{1} \wedge \ldots \wedge d d^{c} u_{n}=o\left(\left(C_{n}(B(a, r))\right)^{\frac{k}{n}}\right) .
$$

The next result should be compared with Theorem 6.3 in 7 .

Theorem 4.5. Let $u_{1}, \ldots, u_{n} \in \mathcal{E}$. Then there exists $\tilde{u} \in \mathcal{E}^{a}$ such that

$$
d d^{c} u_{1} \wedge \ldots \wedge d d^{c} u_{n}=\left(d d^{c} \tilde{u}\right)^{n}+\left.d d^{c} u_{1} \wedge \ldots \wedge d d^{c} u_{n}\right|_{\left\{u_{1}=\ldots=u_{n}=-\infty\right.} .
$$

Proof. First, we write

$$
d d^{c} u_{1} \wedge \ldots \wedge d d^{c} u_{n}=\mu+\left.d d^{c} u_{1} \wedge \ldots \wedge d d^{c} u_{n}\right|_{\left\{u_{1}=\ldots=u_{n}=-\infty\right\}},
$$

where

$$
\mu=\left.d d^{c} u_{1} \wedge \ldots \wedge d d^{c} u_{n}\right|_{\left\{u_{1}>-\infty\right\} \cup \ldots \cup\left\{u_{n}>-\infty\right\}} .
$$

It is easy to see that $\mu \ll C_{n}$ in every $E \Subset \Omega$. Indeed, by Theorem 4.1 we have

$$
\left.d d^{c} u_{1} \wedge \ldots \wedge d d^{c} u_{n}\right|_{\left\{u_{1}>-j\right\}}=\left.d d^{c} \max \left(u_{1},-j\right) \wedge \ldots \wedge d d^{c} u_{n}\right|_{\left\{u_{1}>-j\right\}} .
$$

Hence, by Proposition 4.4 (i) it follows that $\left.d d^{c} u_{1} \wedge \ldots \wedge d d^{c} u_{n}\right|_{\left\{u_{1}>-j\right\}} \ll C_{n}$ in every $E \Subset \Omega$. Next, it remains to show that there exists $\tilde{u} \in \mathcal{E}^{a}$ such that $\mu=\left(d d^{c} \tilde{u}\right)^{n}$. Let $\left\{\Omega_{j}\right\}$ be an increasing exhaustion sequence of $\Omega$. For each $j \geq 1$ put $\mu_{j}=\left.\mu\right|_{\Omega_{j}}$. By [1] there exists $\tilde{u}_{j} \in \mathcal{F}$ such that $\left(d d^{c} \tilde{u}_{j}\right)^{n}=\mu_{j}$. Notice that $\mu_{j} \nearrow \mu$ and

$$
\left(d d^{c} \tilde{u}_{j}\right)^{n} \leq \mu \leq\left(d d^{c}\left(u_{1}+\ldots+u_{n}\right)\right)^{n} .
$$

Applying the comparison principle we obtain

$$
\tilde{u}_{j} \searrow \tilde{u} \geq u_{1}+\ldots+u_{n} \in \mathcal{E} .
$$

Hence, $\tilde{u} \in \mathcal{E}^{a}$ and $\left(d d^{c} \tilde{u}\right)^{n}=\lim _{j \rightarrow \infty}\left(d d^{c} \tilde{u}_{j}\right)^{n}=\mu$. The proof is thereby completed.

Corollary 4.6. $u_{1}, \ldots, u_{n} \in \mathcal{E}$. Then the following are equivalent:

i) $d d^{c} u_{1} \wedge \ldots \wedge d d^{c} u_{n} \ll C_{n}$ in every $E \Subset \Omega$,

ii) $\int_{\left\{u_{1}=\ldots=u_{n}=-\infty\right\}} d d^{c} u_{1} \wedge \ldots \wedge d d^{c} u_{n}=0$,

iii) $\int_{\left\{u_{1}<-s, \ldots, u_{n}<-s\right\} \cap E} d d^{c} u_{1} \wedge \ldots \wedge d d^{c} u_{n} \rightarrow 0$ as $s \rightarrow+\infty$ for all $E \Subset \Omega$.

Proof. The proof is a direct application of Theorem 4.5.

The comparison principle for class $\mathcal{F}$ was studied in [9] and [16], [17]. By using Proposition 3.1 and Theorem 4.1 we prove a Xing-type comparison principle for $\mathcal{F}$. 
Theorem 4.7. Let $u \in \mathcal{F}, v \in \mathcal{E}$ and $1 \leq k \leq n$. Then

$$
\begin{aligned}
& \frac{1}{k !} \int_{\{u<v\}}(v-u)^{k} d d^{c} w_{1} \wedge \ldots \wedge d d^{c} w_{n}+\int_{\{u<v\}}\left(r-w_{1}\right)\left(d d^{c} v\right)^{k} \wedge d d^{c} w_{k+1} \wedge \ldots \wedge d d^{c} w_{n} \\
& \quad \leq \int_{\{u<v\} \cup\{u=v=-\infty\}}\left(r-w_{1}\right)\left(d d^{c} u\right)^{k} \wedge d d^{c} w_{k+1} \wedge \ldots \wedge d d^{c} w_{n}
\end{aligned}
$$

for all $w_{j} \in P S H(\Omega), 0 \leq w_{j} \leq 1, j=1, \ldots, k, w_{k+1}, \ldots, w_{n} \in \mathcal{F}$ and all $r \geq 1$.

Proof. Let $\epsilon>0$. We set $\tilde{v}=\max (u, v-\epsilon)$. By a) in Proposition 3.1 we have

$$
\begin{aligned}
& \frac{1}{k !} \int_{\Omega}(\tilde{v}-u)^{k} d d^{c} w_{1} \wedge \ldots \wedge d d^{c} w_{n}+\int_{\Omega}\left(r-w_{1}\right)\left(d d^{c} \tilde{v}\right)^{k} \wedge d d^{c} w_{k+1} \wedge \ldots \wedge d d^{c} w_{n} \\
& \quad \leq \int_{\Omega}\left(r-w_{1}\right)\left(d d^{c} u\right)^{k} \wedge d d^{c} w_{k+1} \wedge \ldots \wedge d d^{c} w_{n} .
\end{aligned}
$$

Since $\{u<\tilde{v}\}=\{u<v-\epsilon\}$ and using Theorem 4.1 we have

$$
\begin{aligned}
& \frac{1}{k !} \int_{\{u<v-\epsilon\}}(v-\epsilon-u)^{k} d d^{c} w_{1} \wedge \ldots \wedge d d^{c} w_{n} \\
& \quad+\int_{\{u<v-\epsilon\}}\left(r-w_{1}\right)\left(d d^{c} v\right)^{k} \wedge d d^{c} w_{k+1} \wedge \ldots \wedge d d^{c} w_{n} \\
& \quad \leq \int_{\{u \leq v-\epsilon\}}\left(r-w_{1}\right)\left(d d^{c} u\right)^{k} \wedge d d^{c} w_{k+1} \wedge \ldots \wedge d d^{c} w_{n} \\
& \leq \int_{\{u<v\} \cup\{u=v=-\infty\}}\left(r-w_{1}\right)\left(d d^{c} u\right)^{k} \wedge d d^{c} w_{k+1} \wedge \ldots \wedge d d^{c} w_{n} .
\end{aligned}
$$

Letting $\epsilon \searrow 0$ we obtain

$$
\begin{aligned}
& \frac{1}{k !} \int_{\{u<v\}}(v-u)^{k} d d^{c} w_{1} \wedge \ldots \wedge d d^{c} w_{n}+\int_{\{u<v\}}\left(r-w_{1}\right)\left(d d^{c} v\right)^{k} \wedge d d^{c} w_{k+1} \wedge \ldots \wedge d d^{c} w_{n} \\
& \quad \leq \int_{\{u<v\} \cup\{u=v=-\infty\}}\left(r-w_{1}\right)\left(d d^{c} u\right)^{k} \wedge d d^{c} w_{k+1} \wedge \ldots \wedge d d^{c} w_{n} .
\end{aligned}
$$

Corollary 4.8. Let $u \in \mathcal{E}^{a}$ be such that $u \geq v$ for all functions $v \in \mathcal{E}$ satisfying $\left(d d^{c} u\right)^{n} \leq\left(d d^{c} v\right)^{n}$. Then

$$
\begin{aligned}
& \frac{1}{n !} \int_{\{u<v\}}(v-u)^{n} d d^{c} w_{1} \wedge \ldots \wedge d d^{c} w_{n}+\int_{\{u<v\}}\left(r-w_{1}\right)\left(d d^{c} v\right)^{n} \\
& \quad \leq \int_{\{u<v\}}\left(r-w_{1}\right)\left(d d^{c} u\right)^{n}
\end{aligned}
$$

for all $v \in \mathcal{E}, r \geq 1$ and all $w_{1}, \ldots, w_{n} \in P S H(\Omega), 0 \leq w_{1}, \ldots, w_{n} \leq 1$. 
Proof. Let $\left\{\Omega_{j}\right\}$ be an increasing exhaustion sequence of relatively compact subdomains of $\Omega$. Set $\mu_{j}=1_{\Omega_{j}} 1_{\{u>-j\}}\left(d d^{c} u\right)^{n}$, where $1_{E}$ denotes the characteristic function of $E \subset \Omega$. Applying Theorem 4.1 we have

$$
\mu_{j}=1_{\Omega_{j}} 1_{\{u>-j\}}\left(d d^{c} \max (u,-j)\right)^{n} \leq 1_{\Omega_{j}}\left(d d^{c} \max (u,-j)\right)^{n} .
$$

Take $\phi \in \mathcal{E}_{0}(\Omega) \cap C(\bar{\Omega})$. Put

$$
\phi_{j}=\max \left(u,-j, a_{j} \phi\right),
$$

where $a_{j}=\frac{-j}{\sup _{\Omega_{j+1}} \phi}$. Then $\phi_{j}=\max (u,-j)$ on $\Omega_{j+1}, \phi_{j} \in \mathcal{E}_{0}$ and

$$
\mu_{j} \leq 1_{\Omega_{j}}\left(d d^{c} \max (u,-j)\right)^{n}=1_{\Omega_{j}}\left(d d^{c} \phi_{j}\right)^{n} \leq\left(d d^{c} \phi_{j}\right)^{n} .
$$

By Kołodziej's theorem (see [15]) there exists $u_{j} \in \mathcal{E}_{0}$ such that

$$
\left(d d^{c} u_{j}\right)^{n}=\mu_{j}=1_{\Omega_{j}} 1_{\{u>-j\}}\left(d d^{c} u\right)^{n}
$$

for all $j \geq 1$. By the comparison principle we have $u_{j} \searrow \tilde{u} \geq u$. On the other hand, since $\left(d d^{c} u\right)^{n}(\{u=-\infty\})=0$, it follows that

$$
\left(d d^{c} u_{j}\right)^{n}=1_{\Omega_{j}} 1_{\{u>-j\}}\left(d d^{c} u\right)^{n} \rightarrow\left(d d^{c} u\right)^{n}
$$

weakly as $j \rightarrow \infty$. Thus $\left(d d^{c} \tilde{u}\right)^{n}=\lim _{j \rightarrow \infty}\left(d d^{c} u_{j}\right)^{n}=\left(d d^{c} u\right)^{n}$. By the hypothesis we have $\tilde{u}=u$. Applying Theorem 4.7 we get

$$
\begin{aligned}
& \frac{1}{n !} \int_{\left\{u_{j}<v\right\}}\left(v-u_{j}\right)^{n} d d^{c} w_{1} \wedge \ldots \wedge d d^{c} w_{n}+\int_{\left\{u_{j}<v\right\}}\left(r-w_{1}\right)\left(d d^{c} v\right)^{n} \\
& \quad \leq \int_{\left\{u_{j}<v\right\}}\left(r-w_{1}\right)\left(d d^{c} u_{j}\right)^{n} \\
& \quad \leq \int_{\left\{u_{j}<v\right\}}\left(r-w_{1}\right)\left(d d^{c} u\right)^{n} .
\end{aligned}
$$

Letting $j \rightarrow \infty$ we obtain

$$
\frac{1}{n !} \int_{\{u<v\}}(v-u)^{n} d d^{c} w_{1} \wedge \ldots \wedge d d^{c} w_{n}+\int_{\{u<v\}}\left(r-w_{1}\right)\left(d d^{c} v\right)^{n} .
$$

Arguing as in Theorem 4.7 we prove a Xing-type comparison principle for $\mathcal{E}$.

Theorem 4.9. Let $u, v \in \mathcal{E}$ and $1 \leq k \leq n$ be such that $\varliminf_{z \rightarrow \partial \Omega}[u(z)-v(z)] \geq 0$. Then

$$
\begin{gathered}
\frac{1}{k !} \int_{\{u<v\}}(v-u)^{k} d d^{c} w_{1} \wedge \ldots \wedge d d^{c} w_{n} \\
\quad+\int_{\{u<v\}}\left(r-w_{1}\right)\left(d d^{c} v\right)^{k} \wedge d d^{c} w_{k+1} \wedge \ldots \wedge d d^{c} w_{n} \\
\leq \int_{\{u<v\} \cup\{u=v=-\infty\}}\left(r-w_{1}\right)\left(d d^{c} u\right)^{k} \wedge d d^{c} w_{k+1} \wedge \ldots \wedge d d^{c} w_{n} \\
\text { for all } w_{j} \in \operatorname{PSH}(\Omega), 0 \leq w_{j} \leq 1, j=1, \ldots, k, w_{k+1}, \ldots, w_{n} \in \mathcal{E} \text { and all } r \geq 1 .
\end{gathered}
$$


Proof. Let $\epsilon>0$. We set $\tilde{v}=\max (u, v-\epsilon)$. By b) in Proposition 3.1 we have

$$
\begin{aligned}
& \frac{1}{k !} \int_{\Omega}(\tilde{v}-u)^{k} d d^{c} w_{1} \wedge \ldots \wedge d d^{c} w_{n}+\int_{\Omega}\left(r-w_{1}\right)\left(d d^{c} \tilde{v}\right)^{k} \wedge d d^{c} w_{k+1} \wedge \ldots \wedge d d^{c} w_{n} \\
& \quad \leq \int_{\Omega}\left(r-w_{1}\right)\left(d d^{c} u\right)^{k} \wedge d d^{c} w_{k+1} \wedge \ldots \wedge d d^{c} w_{n} .
\end{aligned}
$$

Since $\{u<\tilde{v}\}=\{u<v-\epsilon\}$ and using Theorem 4.1 we have

$$
\begin{aligned}
& \frac{1}{k !} \int_{\{u<v-\epsilon\}}(v-\epsilon-u)^{k} d d^{c} w_{1} \wedge \ldots \wedge d d^{c} w_{n} \\
& \quad+\int_{\{u<v-\epsilon\}}\left(r-w_{1}\right)\left(d d^{c} v\right)^{k} \wedge d d^{c} w_{k+1} \wedge \ldots \wedge d d^{c} w_{n} \\
& \quad \leq \int_{\{u \leq v-\epsilon\}}\left(r-w_{1}\right)\left(d d^{c} u\right)^{k} \wedge d d^{c} w_{k+1} \wedge \ldots \wedge d d^{c} w_{n} \\
& \leq \int_{\{u<v\} \cup\{u=v=-\infty\}}\left(r-w_{1}\right)\left(d d^{c} u\right)^{k} \wedge d d^{c} w_{k+1} \wedge \ldots \wedge d d^{c} w_{n} .
\end{aligned}
$$

Letting $\epsilon \searrow 0$ we obtain

$$
\begin{aligned}
& \frac{1}{k !} \int_{\{u<v\}}(v-u)^{k} d d^{c} w_{1} \wedge \ldots \wedge d d^{c} w_{n}+\int_{\{u<v\}}\left(r-w_{1}\right)\left(d d^{c} v\right)^{k} \wedge d d^{c} w_{k+1} \wedge \ldots \wedge d d^{c} w_{n} \\
& \quad \leq \int_{\{u<v\} \cup\{u=v=-\infty\}}\left(r-w_{1}\right)\left(d d^{c} u\right)^{k} \wedge d d^{c} w_{k+1} \wedge \ldots \wedge d d^{c} w_{n} .
\end{aligned}
$$

\section{ACKNOWLEDGMENT}

We are grateful to Professor Urban Cegrell for useful discussions that helped to improve the paper. We are grateful to Per Åhag for fruitful comments. The authors are also indebted to the referee for his useful comments. This work was supported by the National Research Program for Natural Sciences, Vietnam.

\section{REFERENCES}

[1] P. Åhag, The complex Monge-Ampère operator on bounded hyperconvex domains, Ph.D. Thesis, Umeå University, 2002.

[2] Z. Błocki, On the definition of the Monge-Ampère operator in $\mathbf{C}^{2}$, Math. Ann., 328 (2004), 415-423. MR2036329 (2004m:32074)

[3] Z. Błocki, The domain of definition of the complex Monge-Ampère operator, Amer. J. Math, 128 (2006), 519-530. MR2214901 (2006k:32076)

[4] E. Bedford and B. A.Taylor, The Dirichlet problem for the complex Monge-Ampère operator, Invent. Math. 37 (1976), 1-44. MR0445006 (56:3351)

[5] E. Bedford and B. A.Taylor, A new capacity for plurisubharmonic functions, Acta Math., 149 (1982), 1-40. MR674165 (84d:32024)

[6] E. Bedford and B. A.Taylor, Fine topology, Silov boundary, and $\left(d d^{c}\right)^{n}$, J. Funct. Anal. 72 (1987), 225-251. MR886812 (88g:32033)

[7] U. Cegrell, Pluricomplex energy, Acta Math., 180 (1998), 187-217. MR.1638768|(99h:32016) 
[8] U. Cegrell, The general definition of the complex Monge-Ampère operator, Ann. Inst. Fourier (Grenoble) 54 (2004), 159-179. MR2069125(2005d:32062)

[9] U. Cegrell, A general Dirichlet problem for the complex Monge-Ampère operator, Ann. Polon. Math., 94 (2008), 131-147. MR2438854

[10] U. Cegrell, S. Kołodziej and A. Zeriahi, Subextention of plurisubharmonic functions with weak singularities, Math. Zeit., 250 (2005), 7-22. MR2136402 (2005m:32064)

[11] R. Czyz, Convergence in capacity of the Perron-Bremermann envelope, Michigan Math. J., 53 (2005), 497-509. MR2207203 (2006k:32066)

[12] D. Coman, N. Levenberg and E. A. Poletsky, Quasianalyticity and pluripolarity, J. Amer. Math. Soc., 18 (2005), 239-252. MR2137977 (2006e:32043)

[13] J-P. Demailly, Monge-Ampère operators, Lelong numbers and intersection theory, Complex Analysis and Geometry, Univ. Ser. Math., Plenum, New York, 1993, 115-193. MR.1211880 (94k:32009)

[14] J-P. Demailly, Potential theory in several variables, preprint (1989).

[15] S. Kolodziej, The range of the complex Monge-Ampère operator, II, Indiana Univ. Math. J., 44 (1995), 765-782. MR1375348 (96m:32013)

[16] P. Hiep, A characterization of bounded plurisubharmonic functions, Ann. Polon. Math., 85 (2004), 233-238. MR2181753 (2006g:32051)

[17] P. Hiep, The comparison principle and Dirichlet problem in the class $\mathcal{E}_{p}(f), p>0$, Ann. Polon. Math., 88 (2006), 247-261. MR2260404 (2007f:32048)

[18] P. Lelong, Notions capacitaires et fonctions de Green pluricomplexes dans les espaces de Banach. C.R. Acad. Sci. Paris Ser. Imath., 305:71-76, 1987. MR901138 (89g:46082)

[19] Y. Xing, Continuity of the complex Monge-Ampère operator. Proc. Amer. Math. Soc., 124 (1996), 457-467. MR 1322940 (96d:32015)

[20] Y. Xing, Complex Monge-Ampère measures of pluriharmonic functions with bounded values near the boundary. Canad. J. Math., 52, (2000),1085-1100. MR.1782339 (2001h:32070)

Department of Mathematics, Hanoi University of Education, Dai hoc Su Pham Hanoi, Cau Giay, Hanoi, Vietnam

Department of Mathematics, Hanoi University of Education, Dai hoc Su Pham Hanoi, Cau Giay, Hanoi, Vietnam

E-mail address: phhiep_vn@yahoo.com 